\title{
Megakaryocyte Cytoskeletal Proteins in Platelet Biogenesis and Diseases
}

\author{
Serge Mbiandjeu ${ }^{1} \quad$ Alessandra Balduini $^{1} \quad$ Alessandro Malara $^{1}$ \\ ${ }^{1}$ Department of Molecular Medicine, University of Pavia, Pavia, Italy \\ Address for correspondence Alessandra Balduini, MD, Department of \\ Thromb Haemost 2022;122:666-678. \\ Molecular Medicine, University of Pavia, via Forlanini 6, 27100 Pavia, \\ Italy (e-mail: alessandra.balduini@unipv.it).
}

Abstract
Keywords
- megakaryocyte
- cytoskeleton
- platelets
- bone marrow
- proplatelet formation
- thrombocytopenia

Thrombopoiesis governs the formation of blood platelets in bone marrow by converting megakaryocytes into long, branched proplatelets on which individual platelets are assembled. The megakaryocyte cytoskeleton responds to multiple microenvironmental cues, including chemical and mechanical stimuli, sustaining the platelet shedding. During the megakaryocyte's life cycle, cytoskeletal networks organize cell shape and content, connect them physically and biochemically to the bone marrow vascular niche, and enable the release of platelets into the bloodstream. While the basic building blocks of the cytoskeleton have been studied extensively, new sets of cytoskeleton regulators have emerged as critical components of the dynamic protein network that supports platelet production. Understanding how the interaction of individual molecules of the cytoskeleton governs megakaryocyte behavior is essential to improve knowledge of platelet biogenesis and develop new therapeutic strategies for inherited thrombocytopenias caused by alterations in the cytoskeletal genes.

\section{Introduction}

Megakaryocytes are highly specialized cells that assemble and generate millions of platelets daily. In humans, megakaryocytes are found primarily in bone marrow (less than $1 \%$ of nucleated cells), ${ }^{1}$ while, in mice, megakaryocytes are also present in the spleen and lungs. ${ }^{2}$ During maturation, megakaryocytes increase in size, replicate their DNA content through endomitosis up to 128 -fold, synthesize unique granules, develop a highly invaginated membrane system, and progressively expand their cytoplasmic content of cytoskeletal proteins. ${ }^{3}$ Mature megakaryocytes appear as giant polyploid cells and undergo a complex transformation of the cytoplasm into long branched proplatelets on which individual platelets are assembled. ${ }^{4}$

Megakaryocyte maturation and proplatelet formation depend on dynamic and strictly regulated modifications in the cytoskeleton. Two cytoskeletal polymer systems are primarily present in megakaryocytes: the tubulin cytoskeleton (microtubules [MTs]) and the actin cytoskeleton (actin filaments). Recent genetic analysis in patients and genetic

received

September 23, 2020

accepted after revision

May 21, 2021

published online

July 4, 2021

manipulations in mice have revealed the essential function of new cytoskeleton regulators in megakaryocyte biology. ${ }^{5}$ Research has focused on several classes of regulatory proteins that control the architecture of the networks formed by cytoskeletal polymers, including nucleation-promoting factors (NPFs), which initiate filament formation; capping proteins, which terminate filament growth; depolymerizing factors and severing factors, which disassemble filaments; crosslinkers and stabilizing proteins, which organize and reinforce higher order network structures; and motor proteins. Understanding how these cytoskeleton regulators control megakaryocyte biology and platelet production is essential for defining the mechanisms of thrombocytopenia and developing new in vitro megakaryocyte models for studying platelet-associated disorders. In this review, we discuss state of the art on the role of cytoskeletal proteins and regulatory molecules in the progressive steps of megakaryocyte maturation and platelet biogenesis. In particular, we will focus on the role of cytoskeletal proteins in (1) megakaryocyte maturation, (2) migration in the bone marrow environment, (3) positioning in the vascular niche and

(c) 2021. Thieme. All rights reserved. Georg Thieme Verlag KG,

Rüdigerstraße 14,

70469 Stuttgart, Germany
DOI https://doi.org/ 10.1055/s-0041-1731717. ISSN 0340-6245. 
podosome formation, and (4) proplatelet extension and platelet biogenesis.

\section{Megakaryocyte Maturation}

Megakaryopoiesis is the process by which hematopoietic stem cells (HSCs) differentiate toward the myeloid lineage to generate mature megakaryocytes. ${ }^{6}$

Thrombopoietin (TPO), synthesized by the liver, is the primary regulator of megakaryocyte progenitor (MKP) expansion and differentiation. ${ }^{7}$ TPO stimulates megakaryocyte growth and platelet production by binding the myeloproliferative leukemia protein receptor on HSC and megakaryocyte surface, leading to Janus activated kinase and signal transducer and activator of transcription signaling pathway activation. TPO sustains megakaryocyte maturation in conjunction with other essential cytokines, including interleukin-3 (IL-3), stem cell factor, IL-6, and IL-11. ${ }^{8}$ Pluripotent HSCs generate megakaryocytes through sequential differentiation into a hierarchical series of progenitor cells: myeloid progenitors, common megakaryocyte-erythroid progenitors (MEPs), and MKPs that mature into megakaryocytes releasing platelets. ${ }^{9}$ However, recent studies have suggested that megakaryocytes can differentiate directly from lineage-biased HSCs or multipotent progenitors, bypassing the MEP. $^{10-14}$

During megakaryocyte maturation, a complex interplay between the cytoskeleton and membranes is required to build up a characteristic structure, called demarcation membrane system (DMS), that provides the intracellular membrane reservoir required for successful elongation of proplatelets and platelet production. The sequential steps of DMS biogenesis and its ultrastructural properties have been described by Eckly and colleagues. ${ }^{15}$ Despite this knowledge, the molecular mechanisms underlying DMS onset and the role of DMS-associated cytoskeleton are not fully understood. The glycoprotein Ib-IX-V (GPIb-IX-V) complex ${ }^{16}$ which labels nascent DMS, and the actin cytoskeleton ${ }^{17}$ are critically involved in the formation of DMS during megakaryopoiesis. The GPIb-IX-V complex is the receptor for the von Willebrand factor on the platelet surface and initiates platelet-subendothelium interactions. ${ }^{16}$ However, mouse megakaryocytes lacking GPIb $\alpha$ or GPIb $\beta$ display an abnormal expansion of the intracellular membrane network of the DMS, suggesting that the entire complex is required for the DMS formation in maturing megakaryocytes. ${ }^{16,18,19}$ Bernard-Soulier syndrome (BSS), a rare bleeding disorder characterized by defects of the GPIb-IX-V complex, presents macrothrombocytopenia and prolonged bleeding. Patients with biallelic mutations always develop a severe form with significantly reduced platelet counts, giant platelets, and recurrent episodes of spontaneous bleeding. In contrast, subjects with the monoallelic mutation have milder phenotypes. ${ }^{18,19}$ Megakaryocytes derived from Bernard-Soulier patients show abnormal proplatelet formation in vitro. ${ }^{20,21}$

The exact role of the GPIb-IX-V complex in megakaryocyte maturation and platelet biogenesis is still unknown. One hypothesis is that it may determine the structure of the submembranous actin network through its binding to the intracellular filamin-A (Fln-A). ${ }^{22}$ Fln-A is a multidomain cytoskeletal protein, present in megakaryocytes and platelets, that stabilizes platelet membranes subjected to shear stress and promotes platelet adhesion by linking membrane glycoproteins to the actin cytoskeleton. ${ }^{23,24}$ Filaminopathies $\mathrm{A}$, caused by mutations in the X-linked FLNA gene, are responsible for a broad spectrum of rare diseases, including two main phenotypes, the X-linked dominant form of periventricular nodular heterotopia and the otopalatodigital syndrome spectrum of disorders. ${ }^{25,26}$ FLNA mutations impact megakaryocyte function, determining the release of giant platelets rapidly removed from the circulation by macrophages. ${ }^{24,27,28}$ In addition, platelets show a decreased ability to aggregate and reduced dense granule secretion. In megakaryocytes/platelets, Fln-A can bind the cytoplasmic tail of the $\beta 3$ subunit and negatively regulate activation of the $\alpha I I b \beta 3$ integrin. A dysregulated Fln-A/ $\alpha$ IIb $\beta 3$ interaction in the downregulation of RhoA activity has been proposed as a mechanism of macrothrombocytopenia. ${ }^{29}$

Bin-Amphiphysin-Rvs (BAR)/Fes-CIP4 homology BAR (FBAR) proteins generate tubular membrane invaginations reminiscent of megakaryocyte DMS. PACSIN2 (also called Syndapin 2) is the only BAR/F-BAR protein reported in megakaryocytes/platelets to associate with the cytoskeletal and scaffold protein Fln-A. Begonja et al showed that in mouse megakaryocytes, Fln-A/PACSIN2 interaction is required to regulate membrane tubulation, likely contributing to DMS formation. ${ }^{30}$ Consistently, mice lacking the F-BARcontaining adaptor protein CIP4 (Cdc42 interacting protein 4) develop mild thrombocytopenia, and CIP4 null megakaryocytes show abnormal DMS, a more rigid membrane, and altered proplatelet formation. ${ }^{31}$

Association of F (filamentous) actin with the DMS is an established event in the maturation of DMS before proplatelet emission. ${ }^{17}$ Actin polymerization is powered by the actin-related protein 2/3 (Arp2/3). The Arp2/3 complex comprises seven evolutionarily conserved subunits, which serve as a nucleation core for de novo actin polymerization. On its own, the Arp2/3 complex displays low intrinsic actin nucleation activity and needs to be activated by NPFs, such as Wiskott-Aldrich syndrome (WAS) protein (WASp) and SCAR (suppressor of the cAMP receptor)/WAVE (WASp family verprolin homologous) proteins. Mutations in the WASp gene cause WAS, a rare X-linked immunodeficiency characterized by eczema, thrombocytopenia, and severe, often recurrent, infections. Microthrombocytopenia is the most common finding at diagnosis, although in rare cases; WAS manifests with macrothrombocytopenia. ${ }^{32}$ More than 300 gene mutations have been identified in the WASp gene, leading to impaired WASp protein configuration. Because of the wide range of genetic mutations, the disease itself has phenotypic variability ranging from severe to mild. Mechanisms of thrombocytopenia remain controversial. Various hypotheses on megakaryocyte dysfunctions or abnormal clearance of defective platelets in the periphery have been reported in patients and mouse models that recapitulate the disease. ${ }^{33-35}$ Although WASp-deficient megakaryocytes can produce proplatelets and platelets in vitro, ${ }^{36}$ defective 
platelet production and premature release of platelets into the bone marrow interstitium have been reported. ${ }^{37,38}$

The study of Schulze et al, using both mouse models and cultured megakaryocytes, revealed that during DMS biogenesis, plasma membrane invaginations are driven by mechanical forces generated through F-actin assembly. ${ }^{39}$ The authors demonstrated that the protrusive force for internal DMS migration relies on actin fibers that assemble at the DMS cytoplasmic face in response to phosphatidylinositol 4,5-bisphosphate (PtdIns(4,5)P2) accumulation and stimulation of the actin-nucleating activity of the Arp2/3 complex by WASp. PtdIns(4,5)P2 is a plasma membrane phospholipid with a recognized role in membrane motility functions, including ruffle formation, endocytosis, membrane traffic, and phagocytosis. $^{39}$ In megakaryocytes, PtdIns(4,5)P2 is generated through the enzymatic activity of the lipid kinase PI-5-P-4-kinase $\alpha$ (PIP4K $\alpha$ ) to promote actin polymerization by activating Rho-like GTPases and WASp. ${ }^{39}$ Megakaryocyte cytoskeleton/membrane dynamics are also regulated by phosphatidylinositol 3 monophosphate (PtdIns3P), and its implication in platelet generation/function has been recently reviewed. ${ }^{40}$

Several actin-binding proteins are emerging as critical regulators of megakaryocyte function, ${ }^{41}$ and most of them are involved in the complex generation of the DMS (-Fig. 1A). $\alpha$-Actinin, a member of the actin-crosslinking protein superfamily, contributes to this process by crosslinking actin filaments into bundles. ${ }^{42}$ Mutations in the ACTN1 gene, encoding for $\alpha$-actinin, cause macrothrombocytopenia and bleeding tendency. ${ }^{42-44}$ However, 15 rare monoallelic ACTN1 variants have been identified in patients characterized by thrombocytopenia with normal platelet size in most cases. ${ }^{45}$ In vitro transduction of mouse fetal liver-derived megakaryocytes with disease-associated ACTN1 variants leads to the disruption of the actin-based cytoskeletal structure, resulting in abnormal megakaryocyte cytoplasm organization and defective proplatelet formation. ${ }^{42,44}$ Tropomodulin3 (Tmod3), the unique form of
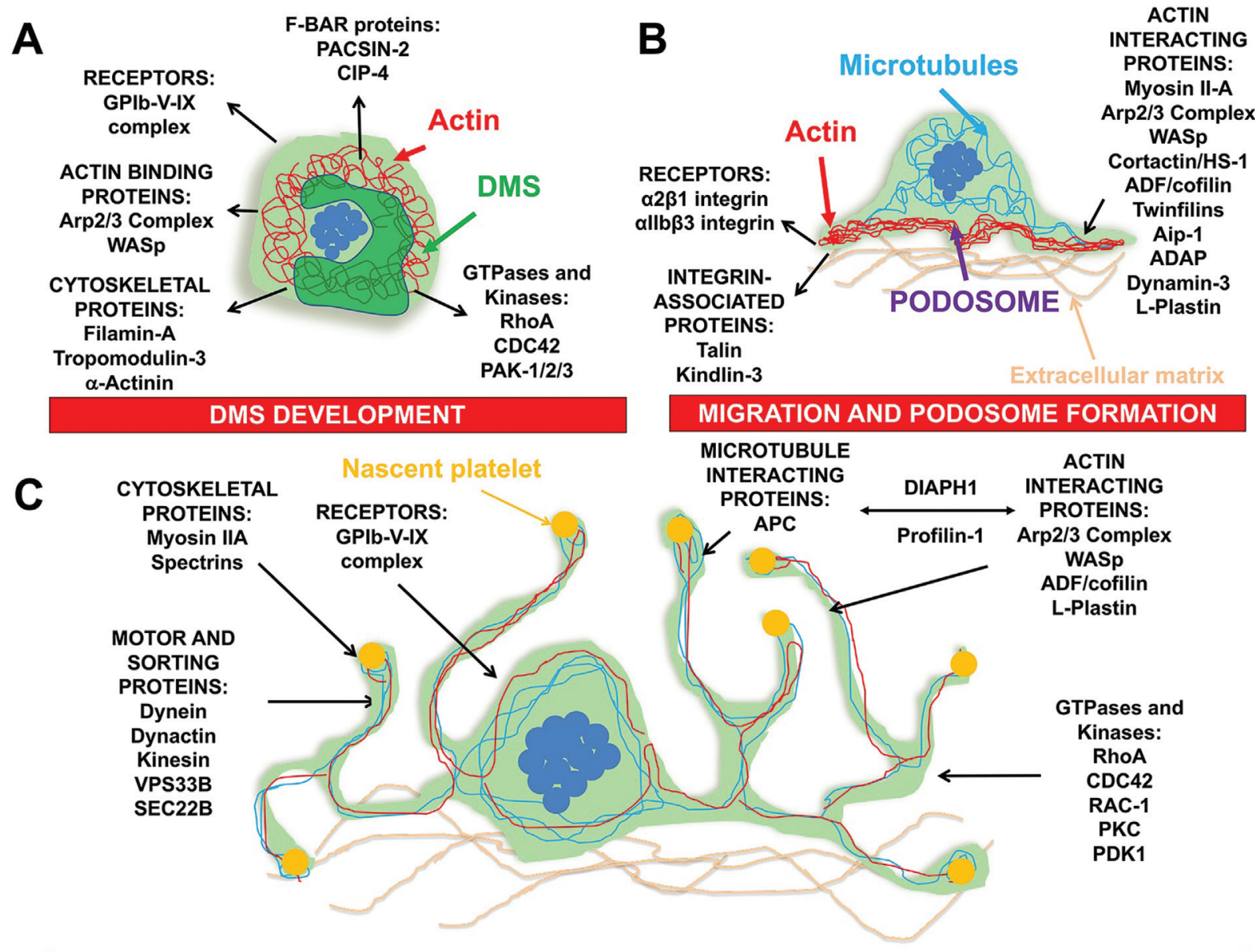

PROPLATELET FORMATION

Fig. 1 List of cytoskeletal proteins and regulators that assist megakaryocyte in the steps toward platelet production. (A) Several cytoskeletal, signaling, and receptor proteins are required to develop the extensive membrane network, known as demarcation membrane system (DMS), during megakaryocyte maturation. (B) Terminally differentiated megakaryocytes migrate and intimately associate with the sinusoidal endothelium of the bone marrow through podosome formation. The coordination of these steps depends on several receptors and actininteracting proteins. (C) Following extensive cytoskeletal remodeling, fully mature megakaryocytes extend cytoplasmic projections called proplatelets into the vessel lumen, where platelets are released under shear forces produced by the circulating blood. Coordination of microtubules or actin dynamics, motor protein functions, GTPase signaling pathways, myosin II-A, and spectrin activity is essential for proplatelet formation and the release of functional platelets. 
tropomodulin expressed in megakaryocytes, is detected in the pointed end of actin filament caps and binds to tropomyosins to promote actin polymerization and stability. Mouse fetal liver Tmod $3^{-1-}$ megakaryocytes show an impairment in cytoplasmic morphogenesis associated with insufficient DMS formation, suggesting an essential role of Tmod3 in the regulation of F-actin organization. ${ }^{46}$ F-actindependent DMS formation requires the activation of the guanosine triphosphatase cell division control protein 42 homolog (Cdc42) and its p21-activated kinase (Pak1/2/3) effectors. Pak1/2/3 are serine/threonine kinases that support cell contractility and survival. ${ }^{47}$ Upon activation by the GTPases, Rac1 and Cdc42, Paks phosphorylate dozens of effector proteins to regulate the mitogen-activated protein kinase signaling and cytoskeletal remodeling. Pharmacological inhibition of Cdc42 and Pak1/2/3 activity promotes the destruction of the DMS and inhibits proplatelet formation. ${ }^{17}$ Genetic deletion of Pak2 in mouse bone marrow is associated with macrothrombocytopenia, decreased platelet half-life, increased megakaryocyte ploidy, and altered microfilament and MT proplatelet structures. ${ }^{48}$ In addition to Paks, Cdc42 regulates the development of the DMS through activated neural-WASp (N-WASp). Chemical knock-down of both Cdc42 and N-WASp in human megakaryocytes determined a structural defect in the DMS and a marked decrease in proplatelet formation. 49

\section{Megakaryocyte Migration in the Bone Marrow}

In the bone marrow, megakaryocytes are closely associated with sinusoids. ${ }^{50,51}$ Megakaryocyte interaction with the microenvironment is essential to guide their maturational chemotaxis toward the vascular niche. Gradients of chemotactic stromal cell-derived factor- $1 \alpha$ (SDF- $1 \alpha)$ and fibroblast growth factor-4 attract megakaryocytes to sinusoidal blood vessels. $^{52,53}$ Although megakaryocytes do not migrate large distances within the bone marrow, ${ }^{51}$ several proteins are involved in the membrane-cytoskeleton rearrangements during megakaryocyte migration (-Fig. 1B). ${ }^{54,55}$ Dynamins (DMNs) are mechanochemical enzymes that participate in membrane dynamics such as cytokinesis, budding of transport vesicles, phagocytosis, and cell motility. ${ }^{56}$ Whereas DMN2 is ubiquitous, both mouse and human megakaryocytes also express DMN3. ${ }^{57}$ DMN3 protein is involved in cellreceptor trafficking during megakaryocyte development and regulates cytoskeleton/membrane dynamics in SDF- $1 \alpha$-induced migration. 54,55

Megakaryocyte migration depends on the interaction of nonmuscle myosin II (NMII) with the actin cytoskeleton. Two types of NMII are expressed in megakaryocytes: nonmuscle myosin heavy chain II-A (NMII-A, MYH9) and nonmuscle myosin heavy chain II-B (NMII-B, MYH10). ${ }^{58}$ NMII-B is expressed in immature megakaryocytes, where it accumulates on the contractile ring in endomitosis transition. NMII$B$ expression is downregulated by Runt-related transcription factor 1 (RUNX1) through MHY10 gene silencing during megakaryocyte polyploidization. This process is essential for switching from mitosis to endomitosis to increase the ploidy level during megakaryocyte differentiation. ${ }^{59,60}$
NMII-A is required for maintaining cell shape and organizing cell cytoplasm. Several studies using megakaryocyte-restricted myosin IIA-deficient mice have reported that NMII-A is involved in the earlier distribution of organelles within megakaryocytes through a mechanism that promotes organelle traveling and tethering onto F-actin cytoskeleton tracks. ${ }^{61,62}$ In addition, inhibition of NMII-A ATPase activity suppresses the SDF- $1 \alpha$-driven migration of the megakaryoblastic Dami cell line. ${ }^{53}$ Pal et al, using both in vitro assays and mouse models with mutated NMII-A, demonstrated that different NMII-A mutations impair megakaryocyte chemotaxis by multiple mechanisms and disrupt megakaryocyte migration toward the vasculature in vivo. ${ }^{63}$

\section{Vascular Niche Positioning and Podosomes Formation} Megakaryocyte interactions with the extracellular environment and positioning in the vascular niche are essential to expand proplatelets and release platelets into the bloodstream. Podosomes are F-actin-rich matrix contacts driven by actin polymerization, initially described in osteoclasts and monocytic cells. ${ }^{64}$ These structures are abundantly present in mouse megakaryocytes in adhesion to extracellular matrices or stimulated with cytokines (e.g., transforming growth factor- $\beta$ or SDF- $1 \alpha) .{ }^{65}$ Although they appear to be individual structures, podosomes are linked to each other in a network on the underlying cytoskeleton, generating superstructures that function as a large unit. The proposed function in megakaryocytes is to sustain extracellular matrix degradation required for cell migration and penetration across the basement membrane of sinusoidal vessels. ${ }^{66}$ Recently, F-actin-based podosome-like structures, called in vivo-megakaryocyte podosomes, have been recognized as a crucial regulatory component in the transendothelial passage of megakaryocyte-derived processes in the native bone marrow environment. ${ }^{67}$

Podosomes' stability is strongly dependent on WASpArp2/3-mediated actin polymerization for proper formation. ${ }^{66}$ Cortactin (Cttn) is an additional core protein of the podosome structure. ${ }^{66,68} \mathrm{Cttn}$ is an F-actin-binding protein that interacts with the actin NPF, the Arp2/3 complex, and stabilizes dynamic branched actin networks. In contrast with Cttn, hematopoietic cell-specific lyn substrate-1 (HS1) is expressed only in hematopoietic lineages. Generation of Cttn/HS1 double knockout mice revealed that these proteins are dispensable for proper podosome assembly, at least in megakaryocytes. ${ }^{69}$

The structure and dynamics of actin filaments are regulated by three phylogenetically distinct classes of actinbinding proteins: ADF/cofilins, Abp1/drebrins, and twinfilins. Members of the $\mathrm{ADF} /$ cofilins are small actin-binding proteins composed of a single actin-depolymerizing factor homology (ADF-H) domain. They bind both actin monomers and filaments and promote rapid filament turnover by depolymerizing/fragmenting actin filaments. Abp1/drebrins are involved in endocytosis, interact only with actin filaments, and do not promote filament depolymerization or fragmentation. Twinfilins only bind actin monomers and localize these monomers, in their "inactive" ADP-form, to 
the sites of rapid actin assembly in cells. ${ }^{70}$ Becker et al dissected the functions of twinfilins and $\mathrm{ADF} /$ cofilin1 in podosome formation in transgenic mouse models. They found a mild reduction in podosome formation in cofilin 1 knockout megakaryocytes adherent to a collagen substrate, whereas twinfilin 1 knockout megakaryocytes seemed to behave mostly as control cells. Double knockout megakaryocytes, lacking both cofilin 1 and twinfilin 1, displayed a dramatic impairment in podosome-like assembly, revealing that members of distinct classes of actin-binding proteins have synergistic effects in the regulation of actin dynamics and podosome formation. ${ }^{71}$

A highly conserved component of the cytoskeleton that cooperates with cofilin in enhancing F-actin disassembly and severing of actin filaments is actin interacting protein 1 (Aip1). Generation of mice with deficiency in the WD40 repeat protein 1 (Wdr1), the mammalian homolog of Aip1, resulted in embryonic lethality, macrothrombocytopenia, and autoinflammatory disease, suggesting that Aip1/cofilin interaction is also essential for megakaryocyte maturation and platelet release. ${ }^{72}$ Consistently, WDR1-related thrombocytopenia was reported in two siblings carrying a WDR1 biallelic mutation associated with autoinflammation, immunodeficiency, and thrombocytopenia. ${ }^{73}$

Additional regulators of actin dynamics during podosome formation include phospholipase D (PLD) and adhesion and degranulation promoting adaptor protein (ADAP). Studies in PLD-deficient megakaryocytes revealed an abnormal actin rearrangement associated with a complete absence of podosome formation. ${ }^{74}$ In contrast, megakaryocytes from ADAP knockout mice display a significant reduction in the number of actin-rich podosomes, altered morphology with signs of fragmentation, and ectopic release of platelet-like particles into the bone marrow compartment. ${ }^{75}$ Mutations in the human FYB gene, encoding for ADAP, results in congenital autosomal recessive small-platelet thrombocytopenia (CARST), a novel autosomal recessive bleeding disorder with small-platelet thrombocytopenia. ${ }^{76}$ So far, two mutations have been identified in the FYB gene (point mutation 393G > A:W131X; 2-bp deletion 1385_1386delAT:Y462), which result in premature stop codons that lead to a truncated gene product or less functional ADAP protein. ${ }^{75,77}$

Integrins in megakaryocyte adhesion and migration: Integrins bind extracellular matrix components and associate with the cell cytoskeleton through various cytoskeletal linker proteins to mechanically connect intracellular and extracellular structures ( $\mathbf{- F i g . ~ 1 B ) ~}{ }^{78}$ Megakaryocytes express abundant levels of integrins essential for their adhesion to proteins of the extracellular matrix in the bone marrow, including collagens, fibronectins, laminins, perlecan, or nidogen. ${ }^{79,80} \alpha 2 \beta 1$ integrin and glycoprotein VI are the primary collagen receptors expressed on megakaryocytes. ${ }^{81}$ In particular, megakaryocyte contact with type I collagen fibrils induces myosin light chain-2 (MLC-2) phosphorylation through the $\alpha 2 \beta 1$ integrin-dependent Rho-ROCK (Rho-associated protein kinase) pathway to regulate the cytoskeleton contractility, cell migration, and platelet release. ${ }^{82-85}$ While, megakaryocyte interactions with fibrinogen, vitronectin, and fibronectin are mediated by $\alpha \mathrm{Ilb} \beta 3$ integrin, which has a pivotal role regulating $\mathrm{F}$-actin cytoskeleton during platelet biogenesis mainly through Fln-A interaction. ${ }^{29,79}$ Mutations in the ITGA2B and ITGB3 genes cause Glanzmann thrombasthenia (GT), a bleeding disorder due to quantitative or qualitative defects of $\alpha$ IIb $\beta 3$ yielding reduced platelet aggregation while maintaining normal platelet count and size. ${ }^{86}$ However, patients with rare autosomal dominant variants of GT with reduced expression and constitutive activation of $\alpha \operatorname{IIb} \beta 3^{86-89}$ present macrothrombocytopenia. The R995Q and R995W mutations in the ITGA2B gene have been reported in GT patients with macrothrombocytopenia. ${ }^{90,91}$ Abnormal proplatelet formation has been detected in megakaryocytes derived from patients with a heterozygous mutation $(2134+1 \mathrm{G}>\mathrm{C})$ of the ITGB3 gene $\mathrm{g}^{92}$ as well as compound heterozygosity for two ITGB3 variants. ${ }^{93}$

Intracellular integrin-associated proteins, such as talin1 and kindlin3, regulate the activation and function of $\alpha 2 \beta 1$ and $\alpha \operatorname{IIb} \beta 3$ integrins during megakaryocyte adhesion. Both kindlin 3 and talin 1 bind to the cytoplasmic tails of integrins, particularly the $\beta 3$ subunit of $\alpha$ Ilb $\beta 3$ integrin, modulating cell spreading or migration. ${ }^{94}$ However, megakaryocyte/platelet-specific talin1-deficient mice have unaltered platelet counts and megakaryocyte localization, excluding a critical role of talin1 in megakaryocyte migration and platelet formation. ${ }^{51,95-97}$ On the contrary, talin1-deficient platelets display a severe hemostatic defect due to impaired $\alpha$ Ilb $\beta 3$ activation, platelet aggregation, and thrombus formation. ${ }^{97}$ To date, specific effects of kindlin-3 on megakaryocyte function have not been reported. Genetic variants of FERMT3 encoding kindlin-3 cause leukocyte adhesion deficiency III (LAD-III) syndrome. ${ }^{98}$ Platelet counts in patients with LAD-III syndrome are normal; however, platelets fail to aggregate, which translates into markedly reduced thrombus formation. ${ }^{99,100}$ This evidence demonstrates that alterations in a cytoskeletal protein may impact platelet and megakaryocyte functions to different extents.

\section{Proplatelet Extension and Platelet Biogenesis}

Proplatelet formation starts with the development and extension of thick pseudopods with an average diameter of 2 to $4 \mu \mathrm{m}$ that are elongated and expanded through repeated cytoskeleton-dependent bending and bifurcation processes, leading to the amplification of these free proplatelet ends. Additional proplatelets can be generated near the primary site of proplatelet formation, ensuring the complete conversion of the megakaryocyte cytoplasm into a complex and extended network of interconnected proplatelets. ${ }^{101}$ The major components of proplatelets are MTs (-Fig. 1C). ${ }^{102,103}$ Three significant factors may contribute to the heterogeneity of MT properties: (1) composition of the $\alpha$ - and $\beta$-tubulin gene-encoded isotypes that are incorporated into MTs; (2) posttranslational modifications of tubulin, and (3) interactions with diverse MT-interacting proteins (MIPs). Humans encode at least seven $\alpha$ and eight $\beta$ tubulin isotypes with distinct expression profiles according to cell identity and stage of development. ${ }^{104} \mathrm{~A}$ hematopoietic-specific tubulin isotype, tubulin $\beta 1$ class VI 
( $\beta 1$ tubulin), is expressed only in mature megakaryocytes and represents $90 \%$ of the total $\beta$-tubulin pool in mature platelets. ${ }^{102,105,106}$ Mutations in the human gene encoding for this tubulin isotype (TUBB1) lead to TUBB1-related thrombocytopenia, primarily due to defective proplatelet formation and abnormal protrusion-like platelet release. ${ }^{107-109}$ The first functional TUBB1 variant reported in humans was the double nucleotide substitution c.128_129delAGinsCC predicting p.Gln43Pro in $\beta 1$-tubulin chain. This common variant is associated with a population of enlarged round platelets with defective marginal bands and abnormally distributed cytoplasmic organelles. ${ }^{110}$ In a collection of patients with monoallelic BSS, an additional common variant TUBB1 c.920G > A predicting p.Arg307His in the $\beta 1$-tubulin chain was associated with a lower platelet count. ${ }^{111}$ The first reported rare TUBB1 variant in humans linked to thrombocytopenia was a heterozygous c.952C $>\mathrm{T}$ predicting a p.Arg318Trp substitution in the tubulin $\beta 1$ chain. This mutation was identified in a patient with macrothrombocytopenia (platelet count: $40-60 \times 10^{9} / \mathrm{L}$ ) but with no bleeding and normal platelet functional responses. ${ }^{108}$ A second rare TUBB1 c.779T > C variant predicting p.Phe260Ser was identified in a further pedigree with mild macrothrombocytopenia (platelet counts: $97-125 \times 10^{9} / \mathrm{L}$ ) but no abnormal bleeding. ${ }^{105,107}$ In recent studies, rare missense or high impact TUBB1 variants have been identified in thrombocytopenic patients using rapid throughput sequencing, ${ }^{112,113}$ whereas novel gene mutations have been detected in patients with thyroid dysgenesis. ${ }^{114}$

In addition to $\beta$-tubulin, expression of $\alpha 1$ isotype increases early during megakaryocyte differentiation. It remains stable until full maturation, while expressions of $\alpha 4$ and $\alpha 8$ transcripts are upregulated at the stage preceding proplatelet extension and marginal band formation. ${ }^{115}$ Moderate macrothrombocytopenia is present in human individuals with naturally occurring mutations of the TUBA4A gene ${ }^{116}$ and in a mouse strain with a missense mutation in the Tuba4a gene, suggesting a crucial role of $\alpha 4 \mathrm{~A}$-tubulin in late stages of megakaryocyte maturation. ${ }^{116}$

Studies in mouse megakaryocytes have reported that in addition to tubulin isotype composition, posttranslational modification patterns of MTs are required for proper platelet release. ${ }^{115}$ Van Dijk et al disclosed that $\beta 1$ tubulin acetylation occurs along the MTs colonizing the extending proplatelet. Its steady-state level increases with elongation kinetics, suggesting that acetylation is required for proplatelet elongation. Polyglutamylation of MTs marks the most dynamic growing region of proplatelets, the swellings, and severed cytoplasts. ${ }^{115}$ MTs assume several functions during proplatelet formation: (1) they generate the driving force of proplatelet elongation; (2) they mediate the transport of granules and organelles into nascent platelets; and (3) they are arranged into a submembranous structure, the marginal band, which encircles the nascent platelets.

MTs drive proplatelet initiation and elongation: proplatelet formation is characterized by repetitive phases of extension (elongation), pause, and retraction of proplatelet shafts. ${ }^{117}$ Proplatelets elongate at an average rate of $0.85 \mu \mathrm{m} / \mathrm{min}$ in an
MT-dependent process. The mechanisms driving proplatelet elongation are the continuous assembly and sliding of MTs. ${ }^{117,118}$ However, treatment with inhibitors of MT polymerization does not modify the rate of proplatelet shaft elongation, suggesting that the sliding of overlapping MTs is a vital component of proplatelet elongation. ${ }^{118}$ Further, dynamic bending and branching processes bifurcate the shaft multiple times and expand the number of free proplatelet ends. In the proplatelet ends, a single MT rolls up into a circumferential coil and maintains the discoid shape of nascent platelets. ${ }^{117}$

MT sliding and organelles transport in proplatelets: MT sliding and transport of organelles and granules into proplatelets are mediated by two cytoplasmic motor proteins: dynein and kinesin. ${ }^{117,119,120}$ Dynein is primarily responsible for MTs sliding in synergy with the cofactor dynactin. At the same time, kinesin localizes with granules and organelles within the proplatelets and provides the motile force that moves cargo over MT into the proplatelet. Vacuolar protein sorting-associated protein 33b (VPS33B) and VPS33B interacting protein (VIPAS39 or VIPAR) are two vesicle-mediated protein-sorting proteins that form a functional complex involved in $\alpha$-granule trafficking and biogenesis. A recent study has identified two novel interactors of this complex, $\alpha$ tubulin and Sec22 vesicle trafficking protein homologue B (SEC22B). This demonstrated that VPS33B expression is required for the transportation of the von Willebrand factor by SEC22B and the $\alpha$-granule, from megakaryocytes to proplatelets. ${ }^{121,122}$ VPS33B-deficient mouse megakaryocytes have normal megakaryocyte maturation and proplatelet formation. ${ }^{123}$ Simultaneously, mutations in human VPS33B and VIPAS39 genes cause arthrogryposis, renal dysfunction, and cholestasis syndrome, a rare disorder associated with several platelet abnormalities. ${ }^{124}$ Affected patients have variable platelet phenotypes: some cases present thrombocytopenia, and others have normal platelet counts but abnormal platelet function. ${ }^{124}$

Regulators of MT assembly and stability: MT dynamics and stability are maintained by a highly regulated tubulin organization as confirmed by studies in primary cultures of mouse megakaryocytes treated with trastuzumab emtansine (T-DM1). ${ }^{125}$ Thon et al demonstrated that T-DM1 is taken up by mouse megakaryocytes, inhibits megakaryocyte differentiation, and disrupts proplatelet formation by inducing abnormal tubulin organization suppressing MT dynamic instability.

Rho GTPases, such as RhoA, Rac1, and Cdc42, are supposed to regulate tubulin stabilization and assembly in MTs during proplatelet formation. ${ }^{126,127}$ Beyond dynein/dynactin and kinesin, various MIPs are supposed to govern MT assembly into a characteristic circular marginal band. Some MIPs are localized at the ends of growing MTs and are called plus-end tracking proteins (+TIPS). Among them, adenomatous polyposis coli (APC) promotes MT polymerization and protects MTs from shrinking. Indeed, APC deficiency in the megakaryocyte lineage induces an increased cell capacity to extend proplatelets. ${ }^{128}$ MARCKS is a protein kinase C (PKC) substrate that, when dephosphorylated, binds to and 
sequesters PI-4,5-P2 at the membrane. Upon phosphorylation, MARCKS relocates from the plasma membrane to internal demarcation membranes. Conversely, dephosphorylation of MARCKS results in its relocation to the plasma membrane and restoration of PI-4,5-P2 binding. Machlus and colleagues demonstrated that inhibition of MARCKS in wildtype mouse megakaryocytes or deletion in MARCKS knockout mice significantly decreased proplatelet formation. This study concluded that MARCKS acts as a "molecular switch" through regulating PI-4,5-P2 signaling to modulate processes like proplatelet extension (MT-driven) versus proplatelet branching (actin polymerization-driven). ${ }^{129}$

An intriguing aspect is the in vivo contribution of tubulin cytoskeleton to platelet release, given that most of the data on the role of MT assembly and dynamics during proplatelet formation have been generated using in vitro culture conditions. A recent study reported that MT rearrangements visualized in megakaryocytes in the bone marrow of living mice differ from the previously described mechanism in cultured megakaryocytes. ${ }^{130}$ In $\beta 1$-tubulin-deficient mice, extending proplatelets' morphology and elongation speed, are normal despite the moderate thrombocytopenia suggesting that proplatelet extension generated in vivo is less MTdependent than the in vitro one. ${ }^{130}$ Thus, the development and application of new in vivo imaging systems will clarify the actual contribution of individual cytoskeletal components to platelet generation in the native and complex bone marrow environment.

Actin cytoskeleton mediates proplatelet bending/branching: Differently from tubulins, the role of actin in proplatelet formation remains unclear. Time-lapsed microscopy analysis of proplatelet formation has revealed that proplatelet ends are amplified in an elaborated mechanism of bending/branching and that $\mathrm{F}$-actin is present throughout proplatelets forming the assemblies required to bend and bifurcate proplatelets. ${ }^{101}$ Inhibition of actin polymerization leads to the formation of abnormal, nonbranched proplatelets. ${ }^{101}$ Consistently, a missense mutation in the $\beta$-actin protein has been linked to thrombocytopenia, immunodeficiency, and mental retardation ( $\beta$-actin-related thrombocytopenia). This mutation leads to substituting the glutamic acid residue at position 364 with lysine (E364K) in an essential profilin-binding domain and other actin-regulatory molecules. At the same time, the polymerization of actin is preserved. ${ }^{131}$

Despite these pieces of evidence, the role actin plays in proplatelet formation remains unknown. The hypothesis is that actin polymerization cooperates with NMII-A to guarantee the generation of contraction forces required for the bending and branching processes. ${ }^{62,132}$ An essential component in actin filament branching is the Arp2/3 complex, which, together with the ADF/cofilin family, is a critical regulator of actin polymerization as described above. The deletion of the Arp2/3 complex in the megakaryocyte lineage in mice causes microthrombocytopenia and premature platelet release in the bone marrow compartment. ${ }^{133}$ Accordingly, human mutations in one of the Arp2/3 complex components, actin-related protein 2/3 complex subunit 1B (ARPC1B), result in complete loss of ARPC1B protein and microthrombocytopenia. ${ }^{134}$
Bender and colleagues were the first to dissect the role of the ADF/cofilin family in platelet biogenesis. There are three highly homologous isoforms of the ADF/cofilin family: ADF, $\mathrm{m}$-cofilin (muscle cofilin), and n-cofilin-1 (nonmuscle cofilin). ADF is expressed in epithelial cells, m-cofilin is restricted to muscle cells, while n-cofilin expression is ubiquitous. Genetic deletion of ADF in mice has no effects on platelet counts and size, whereas mice lacking n-cofilin display moderately reduced platelet counts and increased platelet size. ${ }^{135}$ Double-mutant mice, lacking both ADF and n-cofilin in megakaryocytes, show platelet counts dramatically reduced to less than $5 \%$ of control mice and variable size of circulating platelets, including giant and microparticle-like platelets. ${ }^{135}$

The role of the actin-bundling protein L-plastin has emerged in platelet biogenesis. Overexpression and knockdown studies showed that L-plastin promotes MKP migration while negatively regulating proplatelet formation. ${ }^{136}$ Finally, other examples of actin regulators during proplatelet formation include phosphoinositide-dependent protein kinase- 1 (PDK1) ${ }^{137}$ and PKC $\alpha{ }^{132}$

Actin filaments act as cellular tracks for the movement of myosin molecules to generate contractile forces. Actomyosin fibers are critical for proplatelet formation, although the mechanisms are not entirely understood. Mutations in the human MYH9 gene encoding NM-IIA, which cause MYH9related diseases (MYH9-RD), or deletion of the Myh9 gene in mice induces thrombocytopenia. ${ }^{61,138}$ May-Hegglin anomaly is one of the spectra of MYH9-related disorders that includes Sebastian, Epstein, and Fechtner syndromes, all characterized by macrothrombocytopenia, inclusions of NM-IIA in leukocytes, and a variable risk of developing kidney damage, sensorineural deafness, presenile cataracts, and liver enzyme abnormalities. ${ }^{139}$ The presence and severity of spontaneous bleeding tendency correlate with the degree of thrombocytopenia. Most affected individuals have no spontaneous bleeding or only easy bruising. Around $30 \%$ have spontaneous mucocutaneous bleeding, including epistaxis, gum bleeding, or menorrhagia, while life-threatening bleeding is rare. ${ }^{140}$ In general, affected individuals with pathogenic variants involving the head domain of the NM-IIA protein have more severe thrombocytopenia than those with pathogenic variants affecting the tail domain. ${ }^{140}$ Surprisingly, myosin deficiency or inhibition of its activity has been reported to increase the number of cells extending proplatelets in culture. ${ }^{61,141,142}$ Activation of NMII-A activity through the Rho/ROCK/MLC-2 pathway is supposed to inhibit proplatelets in vitro. ${ }^{142}$ In living mice, NMII-A has been shown to regulate the protrusive and retraction forces during proplatelet extension. ${ }^{130}$ Several mechanisms, including increased cell death, defective proplatelet formation, and premature platelet release in the bone marrow compartment, are supposed to contribute to macrothrombocytopenia in MYH9-RD patients. ${ }^{143,144}$ In this regard, the presence of NM-IIA mutations in both human and mouse megakaryocytes is a requisite for altered proplatelet formation. Megakaryocytes differentiated in vitro from MYH9-RD patients form fewer and defective proplatelets due to an 
excess of actomyosin contractility. ${ }^{143,145}$ Knock-in mice with different Myh9 mutations recapitulate MYH9-RD thrombocytopenia and megakaryocyte isolated from these mice presented defective proplatelet formation in vitro. ${ }^{146}$ Nevertheless, megakaryocytes in the marrow of MYH9 patients appear to develop normally and are slightly elevated in number. ${ }^{147}$ Thus, whether additional steps of megakaryopoiesis are affected in MYH9-RD patients remains to be determined.

The presence of a spectrin-based membrane skeleton also supports actomyosin contractility. Spectrin tetramers in megakaryocytes are composed of nonerythroid ( $\alpha$ II and $\beta I I)$ and erythroid ( $\alpha$ I and $\beta I)$ subunits and play a significant role during proplatelet elaboration and proplatelet-

Table 1 Summary of primary mutations in megakaryocyte-cytoskeletal genes associated with thrombocytopenia

\begin{tabular}{|c|c|c|}
\hline Inherited thrombocytopenia/disease & Mutated cytoskeletal protein (gene) & Megakaryocyte/platelet defects \\
\hline Bernard-Soulier syndrome & $\begin{array}{l}\text { GPIb/IX/V complex } \\
\text { (GP1BA, GP1BB, and } \\
\text { GP9 genes) }\end{array}$ & $\begin{array}{l}\text { Mild thrombocytopenia and prolonged } \\
\text { bleeding time. Megakaryocytes from patients } \\
\text { display an abnormal development of the DMS } \\
\text { and defective proplatelet formation. }\end{array}$ \\
\hline Filaminopathies & Filamin A (FLNA gene) & $\begin{array}{l}\text { Macrothrombocytopenia due to aberrant } \\
\text { proplatelet formation yielding giant platelets, } \\
\text { with enlarged and often absent granules, in } \\
\text { reduced number. }\end{array}$ \\
\hline Wiskott-Aldrich syndrome & WASp (WAS gene) & $\begin{array}{l}\text { Microthrombocytopenia due to megakaryo- } \\
\text { cyte dysfunction and abnormal clearance of } \\
\text { defective platelets in the periphery. Abnor- } \\
\text { mal megakaryocyte migration, podosome } \\
\text { formation, and ectopic platelet release in the } \\
\text { bone marrow have been reported. }\end{array}$ \\
\hline $\begin{array}{l}\text { Autoinflammatory periodic fever, } \\
\text { immunodeficiency, and } \\
\text { thrombocytopenia (PFIT) }\end{array}$ & WDR1 (WDR1 gene) & $\begin{array}{l}\text { Thrombocytopenia. No functional studies } \\
\text { have been performed with patient-derived } \\
\text { megakaryocytes. }\end{array}$ \\
\hline ACTN1-related thrombocytopenia & a-Actinin-1 (ACTN1 gene) & $\begin{array}{l}\text { Mild macrothrombocytopenia with low risk of } \\
\text { bleeding. Abnormal F-actin organization in } \\
\text { megakaryocyte cytoplasm and defective } \\
\text { proplatelet formation. }\end{array}$ \\
\hline $\begin{array}{l}\text { Congenital autosomal recessive } \\
\text { small-platelet thrombocytopenia } \\
\text { (CARST) }\end{array}$ & ADAP (FYB gene) & $\begin{array}{l}\text { Microthrombocytopenia characterized by a } \\
\text { reduced platelet life span, decreased per- } \\
\text { centage of mature megakaryocytes, and ec- } \\
\text { topic release of proplatelet-like particles in } \\
\text { the bone marrow. }\end{array}$ \\
\hline Glanzmann thrombasthenia & $\begin{array}{l}\text { Integrin } \alpha \text { llb } \beta 3 \\
\text { (ITGA2B and ITGB3 genes) }\end{array}$ & $\begin{array}{l}\text { Normal platelet counts but defective platelet } \\
\text { aggregation. Rare ITGB2 and ITGB3 variants } \\
\text { characterized by macrothrombocytopenia } \\
\text { due to defective proplatelet formation have } \\
\text { been reported. }\end{array}$ \\
\hline TUBB1-related thrombocytopenia & $\beta$-1-tubulin (TUBB1 gene) & $\begin{array}{l}\text { Macrothrombocytopenia associated with } \\
\text { defective proplatelet formation and abnor- } \\
\text { mal protrusion-like platelet release due to the } \\
\text { functional deficiency of microtubules. }\end{array}$ \\
\hline$\beta$-Actin-related thrombocytopenia & $\beta$-Actin (ACTB gene) & $\begin{array}{l}\text { Thrombocytopenia. No functional studies } \\
\text { have been performed with patient-derived } \\
\text { megakaryocytes. }\end{array}$ \\
\hline $\begin{array}{l}\text { Immunodeficiency with inflammatory } \\
\text { disease and thrombocytopenia }\end{array}$ & $\begin{array}{l}\text { Subunit of the ARP2/3 complex } \\
\text { (ARPC1B gene) }\end{array}$ & $\begin{array}{l}\text { Microthrombocytopenia. Platelets from } \\
\text { patients display aberrant spreading, while } \\
\text { megakaryocytes show altered proplatelet } \\
\text { formation. }\end{array}$ \\
\hline MYH9-related diseases & Nonmuscle myosin IIA (MYH9 gene) & $\begin{array}{l}\text { Macrothrombocytopenia associated with ab- } \\
\text { normal megakaryocyte migration and defec- } \\
\text { tive proplatelet formation. }\end{array}$ \\
\hline DIAPH1-related diseases & $\begin{array}{l}\text { Diaphanous-related formin } 1 \\
\text { (DIAPH1 gene) }\end{array}$ & $\begin{array}{l}\text { Macrothrombocytopenia due to cytoskeletal } \\
\text { dysfunction in megakaryocyte and defective } \\
\text { proplatelet formation. }\end{array}$ \\
\hline
\end{tabular}

Abbreviation: DNS, deviated nasal septum. 
preplatelet-platelet transitions. ${ }^{148,149}$ Assembly of spectrin subunits into tetramers is required for invaginated membrane system maturation and proplatelet extension. Consistently, expression of a spectrin tetramer-disrupting construct in megakaryocytes rapidly destabilizes proplatelets, causing blebbing and swelling. Spectrin tetramers play a role in stabilizing the "barbell shapes" of the penultimate stage in platelet production. ${ }^{149}$

New connections of actin assembly and MT dynamics during proplatelet formation: The steps toward forming functional platelets depend crucially on an underlying network of dynamic, interconnected actin and MT polymers (-Fig. 1C). The crosstalk between these two cytoskeletal systems is coordinated by regulatory proteins such as formins and profilin-1. Formins promote the elongation of linear actin filaments and play a crucial role in the assembly of cytoskeletal structures such as filopodia, lamellipodia, and stress fibers. In addition, formins have recently been shown to regulate MT dynamics directly. ${ }^{121}$ The expression level of the formins DAAM1 (disheveled associated activator of morphogenesis 1), DIAPH1 (diaphanous-related formin 1, also known as mDia1), and FHOD1 (formin homology 2 domain containing 1 ) increases during megakaryocyte maturation. ${ }^{150}$ Recent studies suggest that DIAPH1 may play different roles on actin and MT cytoskeletons during platelet production: a mechanosensitive regulator of F-actin structures and a coordinator of MT dynamics. ${ }^{151-153}$ DIAPH1 knock-down in cultured human megakaryocytes increases proplatelet formation by increasing tubulin polymerization and stability. Conversely, the expression of a constitutively active DIAPH1 inhibits proplatelet extension, ${ }^{152}$ and genetic variants of mDia1 are linked to macrothrombocytopenia in humans. ${ }^{153,154}$ The DIAPH1-related disorder is characterized by macrothrombocytopenia and hearing loss. The R1213X mutation (a heterozygous truncating mutation in the DIAPH1 gene) results in constitutive activation of DIAPH1 with cytoskeletal defects causing reduced proplatelet formation. ${ }^{153-155}$ Recently, the clinical phenotype and pathogenic variants of DIAPH1-RD have been expanded. ${ }^{156}$

Transgenic mouse models deficient in mDia1, Fhod1, and mDia1/Fhod1 double knockout have been recently analyzed to assess the impact of formins on platelet production and function. mDia1 knockout and mDia1/Fhod1 double knockout mice displayed altered platelet count and platelet size, whereas Fhod1 knockout mice displayed normal platelet count and volume. ${ }^{157}$ A surprising outcome from these mice was the lack of any apparent functional platelet defect. The mechanosensitive action of DIAPH1 during actin polymerization is controlled by the regulatory activity of Profilin1. ${ }^{158}$ Profilin-1 can regulate megakaryocyte MT dynamics, most likely via its interaction with formin proteins highlighting the complex and essential role that formins may have in regulating actin and MTs in megakaryocytes and platelets. In this regard, a biphasic effect of profilin- 1 as a regulator of MT $(+)$-end turnover and critical actin regulatory role has been proposed. Many details of the actin-MT interplay remain to be resolved. Further studies are required to assess how profilin and formins contribute to actin and MT dynamics during platelet generation. ${ }^{159,160}$

\section{Concluding Remarks}

Inherited thrombocytopenias are a genetically heterogeneous group of disorders characterized by a reduced blood platelet count. A subgroup of these disorders, known as congenital macrothrombocytopenia, displays an abnormal production of large platelets associated with a bleeding tendency, ranging from mild to severe. Next-generation sequencing has been used to reveal novel genes implicated in these diseases. ${ }^{161}$ Cytoskeletal proteins play essential roles in thrombopoiesis, and mutations in genes regulating the dynamics of cytoskeletal proteins lead to several inherited thrombocytopenias (-Table 1). The repertoire of cytoskeletal proteins required for megakaryocyte development, migration, podosome formation, and production of functional platelets appears to be expanding quickly, suggesting that it is more significant than previously thought. While past studies on megakaryocyte function focused on the role of primary cytoskeletal polymers, such as actin and tubulin, it is becoming clear that these polymers' performance depends on the orchestrated action of several proteins that progressively play a crucial role in platelet biogenesis. Despite ongoing research toward understanding the functional role of cytoskeletal proteins in megakaryocytes, our knowledge of their contribution to the thrombopoietic process is still incomplete. The generation of new mouse models or in vivo visualization of cytoskeletal dynamics will allow us to understand the complex cytoskeletal networks underlying megakaryopoiesis, platelet biogenesis, and function. This knowledge will improve our understanding of the pathophysiological mechanisms of inherited thrombocytopenias associated with megakaryocyte cytoskeleton dysfunction.

\section{Funding}

This study was supported by Associazione Italiana per la Ricerca sul Cancro (AIRC IG 2016 18700, AIRC; Milano, Italy) and U.S. National Institutes of Health (R01HL134829) to A.B.

Conflict of Interest

None declared.

\section{Acknowledgments}

We apologize to all scientists whose important work could not be cited in this review due to space constraints.

\section{References}

1 Ogawa M. Differentiation and proliferation of hematopoietic stem cells. Blood 1993;81(11):2844-2853

2 Grozovsky R, Giannini S, Falet H, Hoffmeister KM. Regulating billions of blood platelets: glycans and beyond. Blood 2015;126 (16):1877-1884

3 Behnke $\mathrm{O}$. An electron microscope study of the megacaryocyte of the rat bone marrow. I. The development of the demarcation 
membrane system and the platelet surface coat. J Ultrastruct Res 1968;24(05):412-433

4 Patel SR, Hartwig JH, Italiano JE Jr. The biogenesis of platelets from megakaryocyte proplatelets. J Clin Invest 2005;115(12): 3348-3354

5 Ghalloussi D, Dhenge A, Bergmeier W. New insights into cytoskeletal remodeling during platelet production. J Thromb Haemost 2019;17(09):1430-1439

6 de Sauvage FJ, Hass PE, Spencer SD, et al. Stimulation of megakaryocytopoiesis and thrombopoiesis by the c-Mpl ligand. Nature 1994;369(6481):533-538

7 Kaushansky K, Lok S, Holly RD, et al. Promotion of megakaryocyte progenitor expansion and differentiation by the c-Mpl ligand thrombopoietin. Nature 1994;369(6481):568-571

8 Kaushansky K. Lineage-specific hematopoietic growth factors. N Engl J Med 2006;354(19):2034-2045

9 Deutsch VR, Tomer A. Advances in megakaryocytopoiesis and thrombopoiesis: from bench to bedside. Br J Haematol 2013;161 (06):778-793

10 Noetzli LJ, French SL, Machlus KR. New insights into the differentiation of megakaryocytes from hematopoietic progenitors. Arterioscler Thromb Vasc Biol 2019;39(07):1288-1300

11 Sanjuan-Pla A, Macaulay IC, Jensen CT, et al. Platelet-biased stem cells reside at the apex of the haematopoietic stem-cell hierarchy. Nature 2013;502(7470):232-236

12 Carrelha J, Meng Y, Kettyle LM, et al. Hierarchically related lineage-restricted fates of multipotent haematopoietic stem cells. Nature 2018;554(7690):106-111

13 Yamamoto R, Morita Y, Ooehara J, et al. Clonal analysis unveils self-renewing lineage-restricted progenitors generated directly from hematopoietic stem cells. Cell 2013;154(05):1112-1126

14 Rodriguez-Fraticelli AE, Wolock SL, Weinreb CS, et al. Clonal analysis of lineage fate in native haematopoiesis. Nature 2018; 553(7687):212-216

15 Eckly A, Heijnen H, Pertuy F, et al. Biogenesis of the demarcation membrane system (DMS) in megakaryocytes. Blood 2014;123(06): 921-930

16 Poujol C, Ware J, Nieswandt B, Nurden AT, Nurden P. Absence of GPIbalpha is responsible for aberrant membrane development during megakaryocyte maturation: ultrastructural study using a transgenic model. Exp Hematol 2002;30(04):352-360

17 Antkowiak A, Viaud J, Severin S, et al. Cdc42-dependent F-actin dynamics drive structuration of the demarcation membrane system in megakaryocytes. J Thromb Haemost 2016;14(06):1268-1284

18 Strassel C, Eckly A, Léon C, et al. Intrinsic impaired proplatelet formation and microtubule coil assembly of megakaryocytes in a mouse model of Bernard-Soulier syndrome. Haematologica 2009;94(06):800-810

19 Ware J, Russell S, Ruggeri ZM. Generation and rescue of a murine model of platelet dysfunction: the Bernard-Soulier syndrome. Proc Natl Acad Sci U S A 2000;97(06):2803-2808

20 Balduini A, Malara A, Pecci A, et al. Proplatelet formation in heterozygous Bernard-Soulier syndrome type Bolzano. J Thromb Haemost 2009;7(03):478-484

21 Balduini A, Malara A, Balduini CL, Noris P. Megakaryocytes derived from patients with the classical form of Bernard-Soulier syndrome show no ability to extend proplatelets in vitro. Platelets 2011;22(04):308-311

22 Kanaji T, Ware J, Okamura T, Newman PJ. GPIb $\alpha$ regulates platelet size by controlling the subcellular localization of filamin. Blood 2012;119(12):2906-2913

23 Balduini A. New aspects of Filamin A versatility. Blood 2011;118 (08):2029-2030

24 Rosa JP, Raslova H, Bryckaert M. Filamin A: key actor in platelet biology. Blood 2019;134(16):1279-1288

25 Parrini E, Ramazzotti A, Dobyns WB, et al. Periventricular heterotopia: phenotypic heterogeneity and correlation with Filamin A mutations. Brain 2006;129(Pt 7):1892-1906
26 Nurden P, Debili N, Coupry I, et al. Thrombocytopenia resulting from mutations in filamin A can be expressed as an isolated syndrome. Blood 2011;118(22):5928-5937

27 Berrou E, Adam F, Lebret M, et al. Heterogeneity of platelet functional alterations in patients with filamin A mutations. Arterioscler Thromb Vasc Biol 2013;33(01):e11-e18

28 Jurak Begonja A, Hoffmeister KM, Hartwig JH, Falet H. FlnA-null megakaryocytes prematurely release large and fragile platelets that circulate poorly. Blood 2011;118(08):2285-2295

29 Donada A, Balayn N, Sliwa D, et al. Disrupted filamin $A / \alpha_{\mathrm{IIb}} \beta_{3}$ interaction induces macrothrombocytopenia by increasing RhoA activity. Blood 2019;133(16):1778-1788

30 Begonja AJ, Pluthero FG, Suphamungmee W, et al. FlnA binding to PACSIN2 F-BAR domain regulates membrane tubulation in megakaryocytes and platelets. Blood 2015;126(01):80-88

31 Chen Y, Aardema J, Kale S, et al. Loss of the F-BAR protein CIP4 reduces platelet production by impairing membrane-cytoskeleton remodeling. Blood 2013;122(10):1695-1706

32 Bastida JM, Del Rey M, Revilla N, et al. Wiskott-Aldrich syndrome in a child presenting with macrothrombocytopenia. Platelets 2017;28(04):417-420

33 Marathe BM, Prislovsky A, Astrakhan A, Rawlings DJ, Wan JY, Strom TS. Antiplatelet antibodies in WASP(-) mice correlate with evidence of increased in vivo platelet consumption. Exp Hematol 2009;37(11):1353-1363

34 Prislovsky A, Strom TS. Increased uptake by splenic red pulp macrophages contributes to rapid platelet turnover in WASP(-) mice. Exp Hematol 2013;41(09):789-798

35 Prislovsky A, Zeng X, Sokolic RA, et al. Platelets from WAS patients show an increased susceptibility to ex vivo phagocytosis. Platelets 2013;24(04):288-296

36 Haddad E, Cramer E, Rivière C, et al. The thrombocytopenia of Wiskott Aldrich syndrome is not related to a defect in proplatelet formation. Blood 1999;94(02):509-518

37 Ingrungruanglert $\mathrm{P}$, Amarinthnukrowh $\mathrm{P}$, Rungsiwiwut R, et al. Wiskott-Aldrich syndrome iPS cells produce megakaryocytes with defects in cytoskeletal rearrangement and proplatelet formation. Thromb Haemost 2015;113(04):792-805

38 Sabri S, Foudi A, Boukour S, et al. Deficiency in the WiskottAldrich protein induces premature proplatelet formation and platelet production in the bone marrow compartment. Blood 2006;108(01):134-140

39 Schulze H, Korpal M, Hurov J, et al. Characterization of the megakaryocyte demarcation membrane system and its role in thrombopoiesis. Blood 2006;107(10):3868-3875

40 Valet C, Levade M, Bellio M, Caux M, Payrastre B, Severin S. Phosphatidylinositol 3-monophosphate: a novel actor in thrombopoiesis and thrombosis. Res Pract Thromb Haemost 2020;4(04): 491-499

41 Poulter NS, Thomas SG. Cytoskeletal regulation of platelet formation: coordination of F-actin and microtubules. Int J Biochem Cell Biol 2015;66:69-74

42 Kunishima S, Okuno Y, Yoshida K, et al. ACTN1 mutations cause congenital macrothrombocytopenia. Am J Hum Genet 2013; 92(03):431-438

43 Westbury SK, Shoemark DK, Mumford AD. ACTN1 variants associated with thrombocytopenia. Platelets 2017;28(06):625-627

44 Guéguen P, Rouault K, Chen JM, et al. A missense mutation in the alpha-actinin 1 gene (ACTN1) is the cause of autosomal dominant macrothrombocytopenia in a large French family. PLoS One 2013;8(09):e74728

45 Vincenot A, Saultier P, Kunishima S, et al; Actn Study Coinvestigators. Novel ACTN1 variants in cases of thrombocytopenia. Hum Mutat 2019;40(12):2258-2269

46 Sui Z, Nowak RB, Sanada C, Halene S, Krause DS, Fowler VM. Regulation of actin polymerization by tropomodulin-3 controls megakaryocyte actin organization and platelet biogenesis. Blood 2015;126(04):520-530 
47 Bokoch GM. Biology of the p21-activated kinases. Annu Rev Biochem 2003;72:743-781

48 Kosoff RE, Aslan JE, Kostyak JC, et al. Pak2 restrains endomitosis during megakaryopoiesis and alters cytoskeleton organization. Blood 2015;125(19):2995-3005

49 Palazzo A, Bluteau O, Messaoudi K, et al. The cell division control protein 42 -Src family kinase-neural Wiskott-Aldrich syndrome protein pathway regulates human proplatelet formation. J Thromb Haemost 2016;14(12):2524-2535

50 Malara A, Balduini A. Blood platelet production and morphology. Thromb Res 2012;129(03):241-244

51 Stegner D, vanEeuwijk JMM, Angay O, et al. Thrombopoiesis is spatially regulated by the bone marrow vasculature. Nat Commun 2017;8(01):127

52 Avecilla ST, Hattori K, Heissig B, et al. Chemokine-mediated interaction of hematopoietic progenitors with the bone marrow vascular niche is required for thrombopoiesis. Nat Med 2004;10 (01):64-71

53 Pecci A, Bozzi V, Panza E, et al. Mutations responsible for MYH9related thrombocytopenia impair SDF-1-driven migration of megakaryoblastic cells. Thromb Haemost 2011;106(04): 693-704

54 Reems JA, Wang W, Tsubata K, et al. Dynamin 3 participates in the growth and development of megakaryocytes. Exp Hematol 2008;36(12):1714-1727

55 Suraneni PK, Corey SJ, Hession MJ, et al. Dynamins 2 and 3 control the migration of human megakaryocytes by regulating CXCR4 surface expression and ITGB1 activity. Blood Adv 2018;2(23): 3540-3552

56 Ferguson SM, De Camilli P. Dynamin, a membrane-remodelling GTPase. Nat Rev Mol Cell Biol 2012;13(02):75-88

57 Wang W, Gilligan DM, Sun S, Wu X, Reems JA. Distinct functional effects for dynamin 3 during megakaryocytopoiesis. Stem Cells Dev 2011;20(12):2139-2151

58 Badirou I, Pan J, Souquere S, et al. Distinct localizations and roles of non-muscle myosin II during proplatelet formation and platelet release. J Thromb Haemost 2015;13(05):851-859

59 Bluteau D, Glembotsky AC, Raimbault A, et al. Dysmegakaryopoiesis of FPD/AML pedigrees with constitutional RUNX1 mutations is linked to myosin II deregulated expression. Blood 2012; 120(13):2708-2718

60 Lordier L, Bluteau D, Jalil A, et al. RUNX1-induced silencing of non-muscle myosin heavy chain IIB contributes to megakaryocyte polyploidization. Nat Commun 2012;3:717

61 Eckly A, Strassel C, Freund M, et al. Abnormal megakaryocyte morphology and proplatelet formation in mice with megakaryocyte-restricted MYH9 inactivation. Blood 2009;113(14): 3182-3189

62 Pertuy F, Eckly A, Weber J, et al. Myosin IIA is critical for organelle distribution and F-actin organization in megakaryocytes and platelets. Blood 2014;123(08):1261-1269

63 Pal K, Nowak R, Billington N, et al. Megakaryocyte migration defects due to nonmuscle myosin IIA mutations underlie thrombocytopenia in MYH9-related disease. Blood 2020;135(21): 1887-1898

64 Linder S, Kopp P. Podosomes at a glance. J Cell Sci 2005;118(Pt 10):2079-2082

65 Schachtner H, Calaminus SD, Thomas SG, Machesky LM. Podosomes in adhesion, migration, mechanosensing and matrix remodeling. Cytoskeleton (Hoboken) 2013;70(10):572-589

66 Schachtner H, Calaminus SD, Sinclair A, et al. Megakaryocytes assemble podosomes that degrade matrix and protrude through basement membrane. Blood 2013;121(13):2542-2552

67 Eckly A, Scandola C, Oprescu A, et al. Megakaryocytes use in vivo podosome-like structures working collectively to penetrate the endothelial barrier of bone marrow sinusoids. J Thromb Haemost 2020;18(11):2987-3001
68 Daly RJ. Cortactin signalling and dynamic actin networks. Biochem J 2004;382(Pt 1):13-25

69 Thomas SG, Poulter NS, Bem D, Finney B, Machesky LM, Watson SP. The actin binding proteins cortactin and HS1 are dispensable for platelet actin nodule and megakaryocyte podosome formation. Platelets 2017;28(04):372-379

70 Paavilainen VO, Merckel MC, Falck S, et al. Structural conservation between the actin monomer-binding sites of twinfilin and actin-depolymerizing factor (ADF)/cofilin. J Biol Chem 2002;277 (45):43089-43095

71 Becker IC, Scheller I, Wackerbarth LM, et al. Actin/microtubule crosstalk during platelet biogenesis in mice is critically regulated by Twinfilin1 and Cofilin1. Blood Adv 2020;4(10):2124-2134

72 Kile BT, Panopoulos AD, Stirzaker RA, et al. Mutations in the cofilin partner Aip1/Wdr1 cause autoinflammatory disease and macrothrombocytopenia. Blood 2007;110(07):2371-2380

73 Standing AS, Malinova D, Hong Y, et al. Autoinflammatory periodic fever, immunodeficiency, and thrombocytopenia (PFIT) caused by mutation in actin-regulatory gene WDR1. J Exp Med 2017;214(01):59-71

74 Stritt S, Thielmann I, Dütting S, Stegner D, Nieswandt B. Phospholipase D is a central regulator of collagen I-induced cytoskeletal rearrangement and podosome formation in megakaryocytes. J Thromb Haemost 2014;12(08):1364-1371

75 Spindler M, van Eeuwijk JMM, Schurr Y, et al. ADAP deficiency impairs megakaryocyte polarization with ectopic proplatelet release and causes microthrombocytopenia. Blood 2018;132 (06):635-646

76 Levin C, Koren A, Pretorius E, et al. Deleterious mutation in the FYB gene is associated with congenital autosomal recessive small-platelet thrombocytopenia. J Thromb Haemost 2015;13 (07):1285-1292

77 Hamamy H, Makrythanasis P, Al-Allawi N, Muhsin AA, Antonarakis SE. Recessive thrombocytopenia likely due to a homozygous pathogenic variant in the FYB gene: case report. BMC Med Genet 2014; $15: 135$

78 Schwartz MA. Integrins and extracellular matrix in mechanotransduction. Cold Spring Harb Perspect Biol 2010;2(12):a005066

79 Larson MK, Watson SP. Regulation of proplatelet formation and platelet release by integrin alpha IIb beta3. Blood 2006;108(05): 1509-1514

80 Balduini A, Pallotta I, Malara A, et al. Adhesive receptors, extracellular proteins and myosin IIA orchestrate proplatelet formation by human megakaryocytes. J Thromb Haemost 2008;6 (11):1900-1907

81 Lagrue-Lak-Hal AH, Debili N, Kingbury G, et al. Expression and function of the collagen receptor GPVI during megakaryocyte maturation. J Biol Chem 2001;276(18):15316-15325

82 Sabri S, Jandrot-Perrus M, Bertoglio J, et al. Differential regulation of actin stress fiber assembly and proplatelet formation by alpha2beta1 integrin and GPVI in human megakaryocytes. Blood 2004;104(10):3117-3125

83 Malara A, Gruppi C, Pallotta I, et al. Extracellular matrix structure and nano-mechanics determine megakaryocyte function. Blood 2011;118(16):4449-4453

84 Semeniak D, Faber K, Öftering P, Manukjan G, Schulze H. Impact of Itga2-Gp6-double collagen receptor deficient mice for bone marrow megakaryocytes and platelets. PLoS One 2019;14(08): e0216839

85 Semeniak D, Kulawig R, Stegner D, et al. Proplatelet formation is selectively inhibited by collagen type I through Syk-independent GPVI signaling. J Cell Sci 2016;129(18):3473-3484

86 Nurden AT, Pillois X. ITGA2B and ITGB3 gene mutations associated with Glanzmann thrombasthenia. Platelets 2018;29(01): 98-101

87 Guillet B, Bayart S, Pillois X, Nurden P, Caen JP, Nurden AT. A Glanzmann thrombasthenia family associated with a TUBB1- 
related macrothrombocytopenia. J Thromb Haemost 2019;17 (12):2211-2215

88 Bury L, Falcinelli E, Chiasserini D, Springer TA, Italiano JE Jr, Gresele P. Cytoskeletal perturbation leads to platelet dysfunction and thrombocytopenia in variant forms of Glanzmann thrombasthenia. Haematologica 2016;101(01):46-56

89 Hauschner H, Mor-Cohen R, Messineo S, et al. Abnormal cytoplasmic extensions associated with active $\alpha$ IIb $\beta 3$ are probably the cause for macrothrombocytopenia in Glanzmann thrombasthenia-like syndrome. Blood Coagul Fibrinolysis 2015;26(03): 302-308

90 Hardisty R, Pidard D, Cox A, et al. A defect of platelet aggregation associated with an abnormal distribution of glycoprotein IIb-IIIa complexes within the platelet: the cause of a lifelong bleeding disorder. Blood 1992;80(03):696-708

91 Kunishima S, Kashiwagi H, Otsu M, et al. Heterozygous ITGA2B R995W mutation inducing constitutive activation of the $\alpha \operatorname{Ilb} \beta 3$ receptor affects proplatelet formation and causes congenital macrothrombocytopenia. Blood 2011;117(20):5479-5484

92 Bury L, Malara A, Gresele P, Balduini A. Outside-in signalling generated by a constitutively activated integrin $\alpha \operatorname{IIb} \beta 3$ impairs proplatelet formation in human megakaryocytes. PLoS One 2012;7(04):e34449

93 Bury L, Zetterberg E, Leinøe EB, et al. A novel variant Glanzmann thrombasthenia due to co-inheritance of a loss- and a gain-offunction mutation of ITGB3: evidence of a dominant effect of gainof-function mutations. Haematologica 2018;103(06):e259-e263

94 Ye F, Snider AK, Ginsberg MH. Talin and kindlin: the one-two punch in integrin activation. Front Med 2014;8(01):6-16

95 Petrich BG, Marchese P, Ruggeri ZM, et al. Talin is required for integrin-mediated platelet function in hemostasis and thrombosis. J Exp Med 2007;204(13):3103-3111

96 Wang Y, Litvinov RI, Chen X, et al. Loss of PIP5KIgamma, unlike other PIP5KI isoforms, impairs the integrity of the membrane cytoskeleton in murine megakaryocytes. J Clin Invest 2008;118 (02):812-819

97 Nieswandt B, Moser M, Pleines I, et al. Loss of talin1 in platelets abrogates integrin activation, platelet aggregation, and thrombus formation in vitro and in vivo. J Exp Med 2007;204(13): 3113-3118

98 Svensson L, Howarth K, McDowall A, et al. Leukocyte adhesion deficiency-III is caused by mutations in KINDLIN3 affecting integrin activation. Nat Med 2009;15(03):306-312

99 Jurk K, Schulz AS, Kehrel BE, et al. Novel integrin-dependent platelet malfunction in siblings with leukocyte adhesion deficiency-III (LAD-III) caused by a point mutation in FERMT3. Thromb Haemost 2010;103(05):1053-1064

100 Nagy M, Mastenbroek TG, Mattheij NJA, et al. Variable impairment of platelet functions in patients with severe, genetically linked immune deficiencies. Haematologica 2018;103(03): 540-549

101 Italiano JE Jr, Lecine P, Shivdasani RA, Hartwig JH. Blood platelets are assembled principally at the ends of proplatelet processes produced by differentiated megakaryocytes. J Cell Biol 1999;147 (06):1299-1312

102 Thon JN, Montalvo A, Patel-Hett S, et al. Cytoskeletal mechanics of proplatelet maturation and platelet release. J Cell Biol 2010; 191(04):861-874

103 Davis B, Toivio-Kinnucan M, Schuller S, Boudreaux MK. Mutation in beta1-tubulin correlates with macrothrombocytopenia in Cavalier King Charles Spaniels. J Vet Intern Med 2008;22(03):540-545

104 Ludueña RF. A hypothesis on the origin and evolution of tubulin. Int Rev Cell Mol Biol 2013;302:41-185

105 Matsumura T, Nakamura-Ishizu A, Takaoka K, et al. TUBB1 dysfunction in inherited thrombocytopenia causes genome instability. Br J Haematol 2019;185(05):888-902

106 Wang D, Villasante A, Lewis SA, Cowan NJ. The mammalian beta-tubulin repertoire: hematopoietic expression of a novel, heterologous beta-tubulin isotype. J Cell Biol 1986;103(05): 1903-1910

107 Kunishima S, Nishimura S, Suzuki H, Imaizumi M, Saito H. TUBB1 mutation disrupting microtubule assembly impairs proplatelet formation and results in congenital macrothrombocytopenia. Eur J Haematol 2014;92(04):276-282

108 Kunishima S, Kobayashi R, Itoh TJ, Hamaguchi M, Saito H. Mutation of the beta1-tubulin gene associated with congenital macrothrombocytopenia affecting microtubule assembly. Blood 2009;113(02):458-461

109 Burley K, Westbury SK, Mumford AD. TUBB1 variants and human platelet traits. Platelets 2018;29(02):209-211

110 Freson K, De Vos R, Wittevrongel C, et al. The TUBB1 Q43P functional polymorphism reduces the risk of cardiovascular disease in men by modulating platelet function and structure. Blood 2005;106(07):2356-2362

111 Basciano PA, Matakas J, Pecci A, et al. $\beta-1$ tubulin R307H SNP alters microtubule dynamics and affects severity of a hereditary thrombocytopenia. J Thromb Haemost 2015;13(04):651-659

112 Bastida JM, Lozano ML, Benito R, et al. Introducing high-throughput sequencing into mainstream genetic diagnosis practice in inherited platelet disorders. Haematologica 2018;103(01): 148-162

113 Johnson B, Lowe GC, Futterer J, et al; UK GAPP Study Group. Whole exome sequencing identifies genetic variants in inherited thrombocytopenia with secondary qualitative function defects. Haematologica 2016;101(10):1170-1179

114 Stoupa A, Adam F, Kariyawasam D, et al. TUBB1 mutations cause thyroid dysgenesis associated with abnormal platelet physiology. EMBO Mol Med 2018;10(12):e9569

115 van Dijk J, Bompard G, Cau J, et al. Microtubule polyglutamylation and acetylation drive microtubule dynamics critical for platelet formation. BMC Biol 2018;16(01):116

116 Strassel C, Magiera MM, Dupuis A, et al. An essential role for $\alpha 4 A-$ tubulin in platelet biogenesis. Life Sci Alliance 2019;2(01): e201900309

117 Bender M, Thon JN, Ehrlicher AJ, et al. Microtubule sliding drives proplatelet elongation and is dependent on cytoplasmic dynein. Blood 2015;125(05):860-868

118 Patel SR, Richardson JL, Schulze H, et al. Differential roles of microtubule assembly and sliding in proplatelet formation by megakaryocytes. Blood 2005;106(13):4076-4085

119 Italiano JE Jr, Patel-Hett S, Hartwig JH. Mechanics of proplatelet elaboration. J Thromb Haemost 2007;5(Suppl 1):18-23

120 Richardson JL, Shivdasani RA, Boers C, Hartwig JH, Italiano JE Jr. Mechanisms of organelle transport and capture along proplatelets during platelet production. Blood 2005;106(13):4066-4075

121 Rogerson C, Gissen P. The CHEVI tethering complex: facilitating special deliveries. J Pathol 2016;240(03):249-252

122 Dai J, Lu Y, Wang C, et al. Vps33b regulates Vwf-positive vesicular trafficking in megakaryocytes. J Pathol 2016;240(01):108-119

123 Bem D, Smith H, Banushi B, et al. VPS33B regulates protein sorting into and maturation of $\alpha$-granule progenitor organelles in mouse megakaryocytes. Blood 2015;126(02):133-143

124 Gissen P, Tee L, Johnson CA, et al. Clinical and molecular genetic features of ARC syndrome. Hum Genet 2006;120(03):396-409

125 Thon JN, Devine MT, Jurak Begonja A, Tibbitts J, Italiano JE Jr. High-content live-cell imaging assay used to establish mechanism of trastuzumab emtansine (T-DM1)-mediated inhibition of platelet production. Blood 2012;120(10):1975-1984

126 Pleines I, Dütting S, Cherpokova D, et al. Defective tubulin organization and proplatelet formation in murine megakaryocytes lacking Rac1 and Cdc42. Blood 2013;122(18):3178-3187

127 Aslan JE, McCarty OJ. Rac and Cdc42 team up for platelets. Blood 2013;122(18):3096-3097

128 Strassel C, Moog S, Mallo L, et al. Microtubule plus-end tracking Adenopolyposis Coli negatively regulates proplatelet formation. Sci Rep 2018;8(01):15808 
129 Machlus KR, Wu SK, Stumpo DJ, et al. Synthesis and dephosphorylation of MARCKS in the late stages of megakaryocyte maturation drive proplatelet formation. Blood 2016;127(11): $1468-1480$

130 Bornert A, Boscher J, Pertuy F, et al. Cytoskeletal-based mechanisms differently regulate in vivo and in vitro proplatelet formation. Haematologica 2021;106(05):1368-1380

131 Nunoi H, Yamazaki T, Tsuchiya H, et al. A heterozygous mutation of beta-actin associated with neutrophil dysfunction and recurrent infection. Proc Natl Acad Sci U S A 1999;96(15):8693-8698

132 Rojnuckarin P, Kaushansky K. Actin reorganization and proplatelet formation in murine megakaryocytes: the role of protein kinase calpha. Blood 2001;97(01):154-161

133 Paul DS, Casari C, Wu C, et al. Deletion of the Arp2/3 complex in megakaryocytes leads to microthrombocytopenia in mice. Blood Adv 2017;1(18):1398-1408

134 Kahr WH, Pluthero FG, Elkadri A, et al. Loss of the Arp2/3 complex component ARPC1B causes platelet abnormalities and predisposes to inflammatory disease. Nat Commun 2017;8:14816

135 Bender M, Eckly A, Hartwig JH, et al. ADF/n-cofilin-dependent actin turnover determines platelet formation and sizing. Blood 2010;116(10):1767-1775

136 Bhatlekar S, Manne BK, Basak I, et al. miR-125a-5p regulates megakaryocyte proplatelet formation via the actin-bundling protein L-plastin. Blood 2020;136(15):1760-1772

137 Geue S, Aurbach K, Manke MC, et al. Pivotal role of PDK1 in megakaryocyte cytoskeletal dynamics and polarization during platelet biogenesis. Blood 2019;134(21):1847-1858

138 Seri M, Pecci A, Di Bari F, et al. MYH9-related disease: MayHegglin anomaly, Sebastian syndrome, Fechtner syndrome, and Epstein syndrome are not distinct entities but represent a variable expression of a single illness. Medicine (Baltimore) 2003;82(03):203-215

139 Pecci A, Ma X, Savoia A, Adelstein RS. MYH9: Structure, functions and role of non-muscle myosin IIA in human disease. Gene 2018; 664:152-167

140 Pecci A, Klersy C, Gresele P, et al. MYH9-related disease: a novel prognostic model to predict the clinical evolution of the disease based on genotype-phenotype correlations. Hum Mutat 2014;35 (02):236-247

141 Chen Z, Naveiras O, Balduini A, et al. The May-Hegglin anomaly gene MYH9 is a negative regulator of platelet biogenesis modulated by the Rho-ROCK pathway. Blood 2007;110(01):171-179

142 Chang Y, Auradé F, Larbret F, et al. Proplatelet formation is regulated by the Rho/ROCK pathway. Blood 2007;109(10): 4229-4236

143 Pecci A, Malara A, Badalucco S, et al. Megakaryocytes of patients with MYH9-related thrombocytopenia present an altered proplatelet formation. Thromb Haemost 2009;102(01):90-96

144 Eckly A, Rinckel JY, Laeuffer P, et al. Proplatelet formation deficit and megakaryocyte death contribute to thrombocytopenia in Myh9 knockout mice. J Thromb Haemost 2010;8(10):2243-2251

145 Chen Y, Boukour S, Milloud R, et al. The abnormal proplatelet formation in MYH9-related macrothrombocytopenia results from an increased actomyosin contractility and is rescued by myosin IIA inhibition. J Thromb Haemost 2013;11(12):2163-2175
146 Zhang Y, Conti MA, Malide D, et al. Mouse models of MYH9related disease: mutations in nonmuscle myosin II-A. Blood 2012;119(01):238-250

147 Heynen MJ, Blockmans D, Verwilghen RL, Vermylen J. Congenital macrothrombocytopenia, leucocyte inclusions, deafness and proteinuria: functional and electron microscopic observations on platelets and megakaryocytes. Br J Haematol 1988;70(04): 441-448

148 Patel-Hett S, Wang H, Begonja AJ, et al. The spectrin-based membrane skeleton stabilizes mouse megakaryocyte membrane systems and is essential for proplatelet and platelet formation. Blood 2011;118(06):1641-1652

149 Thon JN, Macleod H, Begonja AJ, et al. Microtubule and cortical forces determine platelet size during vascular platelet production. Nat Commun 2012;3:852

150 Thomas SG, Calaminus SD, Machesky LM, Alberts AS, Watson SP. G-protein coupled and ITAM receptor regulation of the formin FHOD1 through Rho kinase in platelets. J Thromb Haemost 2011; 9(08):1648-1651

151 Zuidscherwoude M, Green HLH, Thomas SG. Formin proteins in megakaryocytes and platelets: regulation of actin and microtubule dynamics. Platelets 2019;30(01):23-30

152 Pan J, Lordier L, Meyran D, et al. The formin DIAPH1 (mDia1) regulates megakaryocyte proplatelet formation by remodeling the actin and microtubule cytoskeletons. Blood 2014;124(26): 3967-3977

153 Stritt S, Nurden P, Turro E, et al; BRIDGE-BPD Consortium. A gain-of-function variant in DIAPH1 causes dominant macrothrombocytopenia and hearing loss. Blood 2016;127(23): 2903-2914

154 Ganaha A, Kaname T, Shinjou A, et al. Progressive macrothrombocytopenia and hearing loss in a large family with DIAPH1 related disease. Am J Med Genet A 2017;173(10):2826-2830

155 Neuhaus C, Lang-Roth R, Zimmermann U, et al. Extension of the clinical and molecular phenotype of DIAPH1-associated autosomal dominant hearing loss (DFNA1). Clin Genet 2017;91(06): 892-901

156 Westbury SK, Downes K, Burney C, et al; NIHR BioResource-Rare Diseases. Phenotype description and response to thrombopoietin receptor agonist in DIAPH1-related disorder. Blood Adv 2018; 2(18):2341-2346

157 Zuidscherwoude M, Haining EJ, Simms VA, et al. Loss of mDia1 and Fhod1 impacts platelet formation but not platelet function. Platelets 2020;27:1-12

158 Kubota H, Miyazaki M, Ogawa T, Shimozawa T, Kinosita K Jr, Ishiwata S. Biphasic effect of profilin impacts the formin mDia1 force-sensing mechanism in actin polymerization. Biophys J 2017;113(02):461-471

159 Nejedla M, Sadi S, Sulimenko V, et al. Profilin connects actin assembly with microtubule dynamics. Mol Biol Cell 2016;27 (15):2381-2393

160 Rotty JD, Wu C, Haynes EM, et al. Profilin-1 serves as a gatekeeper for actin assembly by Arp2/3-dependent and -independent pathways. Dev Cell 2015;32(01):54-67

161 Savoia A. Molecular basis of inherited thrombocytopenias: an update. Curr Opin Hematol 2016;23(05):486-492 